Research Article

\title{
Fluent Numerical Study of Lifting Resistance and Wave Impact Height of a Cross-Sea Bridge Based on Cloud Computing
}

\author{
Jun $\mathrm{Hu} \mathbb{i D}^{1,2}$ and Jiayu Yu $\mathbf{u}^{1,2}$ \\ ${ }^{1}$ State Key Laboratory of Mountain Bridge and Tunnel Engineering, Chongqing Jiaotong University, Chongqing 400074, China \\ ${ }^{2}$ School of Civil Engineering, Chongqing Jiaotong University, Chongqing 400074, China \\ Correspondence should be addressed to Jun Hu; hujun@cqjtu.edu.cn
}

Received 10 December 2020; Revised 7 January 2021; Accepted 1 March 2021; Published 13 March 2021

Academic Editor: Sang-Bing Tsai

Copyright ( $) 2021$ Jun $\mathrm{Hu}$ and Jiayu Yu. This is an open access article distributed under the Creative Commons Attribution License, which permits unrestricted use, distribution, and reproduction in any medium, provided the original work is properly cited.

\begin{abstract}
Due to the relationship between the elevation of the roads on both sides of the strait and the adjustment with the surrounding landscape, the elevation of the deck of the bridge is restricted when the bridge is designed. When the water level rises below the superstructure of the sea-crossing bridge, the main guardrail of the bridge may be partially or completely submerged in the water, and the superstructure of the bridge is affected by huge waves, which threatens the safety of the bridge. Based on cloud computing technology, this paper establishes a digital model of the crossing bridge and monitors the specific state of the bridge through the simulation of the bridge, collects various data of the bridge, analyzes the dataset, and analyzes the crossing bridge. This paper analyzes the correlation between the magnitude of the wave force and the height of the wave impact on the bridge superstructure and studies the relationship between the lift resistance coefficient and the impact height. The analysis of the lift coefficient and drag coefficient of different water depths in the case of 20 -year operation shows that the height of the main beam where the large wave force acts has two values, 2.0 and $3.0 \mathrm{~m}$ in the case of 20 -year operation; in case of 50 -year working conditions, it is 2.5 and $3.0 \mathrm{~m}$; in case of 100-year working conditions, the lift coefficient is 1.5 and $3.5 \mathrm{~m}$, and the resistance coefficient is 2.0 and $3.5 \mathrm{~m}$. Under the three water depth wave conditions, the fluctuation range of the lifting resistance curve is large when the water level depth is $10 \mathrm{~m}$. Once a large wave occurs, the wave will not spread well due to the shallow current, which will cause a large wave force impact on the bridge. Therefore, when choosing a bridge, it is not recommended to build under a water depth of about $10 \mathrm{~m}$. Optimizing the design of the main beam according to the analysis results of this paper will help to improve the ability of the bridge main beam to resist wave loading and provide a reference for seeking effective measures to reduce the impact of waves.
\end{abstract}

\section{Introduction}

With the continuous increase of bridge spans and vehicle loads, the impact of vehicles on bridges has attracted more and more attention. However, the previous research on the impact of vehicles is mainly on small- and medium-sized bridges. This paper aims to make a qualitative analysis of the factors affecting the impact coefficient of a long-span bridge through numerical simulation.

When the water level below the main beam of a seacrossing bridge becomes high, the main beam may be partially or completely submerged in the water, and the superstructure of the bridge will be impacted by a huge wave force, which will adversely affect the safety and reliability of the bridge [1]. If the correlation between the external load on the bridge superstructure and the height of the wave impact can be accurately estimated, it will be of great significance for the optimal design of the bridge structure and the evaluation of the degree of damage caused by the wave impact [2]. The numerical wave flume is convenient for modeling, saves time and effort in calculation, and can realize simulation analysis of various models. In recent years, it is often used in engineering practice. Numerical wave flume uses numerical calculations to simulate experiments in a physical wave flume. On the one hand, it is necessary to numerically simulate the generation of waves; on the other hand, it is necessary to numerically solve the interaction between the wave and the experimental model. Numerical wave flume 
can guide and partially replace the test of physical wave flume [3]. Due to the complexity of this type of problem, most of the practical applications are studied by model tests. However, the model test is time-consuming and labor-intensive and has a large investment, and it is difficult to make the model completely similar to the real thing, and many details cannot be studied in depth $[4,5]$. With the development and application of Computational Fluid Dynamics (CFD) in the marine engineering field, people pay more and more attention to the application of mathematical models and began to use the widely used Fluent fluid computing software for theoretical research and engineering simulation calculations $[6,7]$.

Mica Grujicic believes that channel beams are widely accepted in engineering due to their lower construction depth and prevention of traffic noise pollution, but they are mostly used for simply supported beam bridges with spans typically less than $30 \mathrm{~m}$. On the railway line from Nanjing to Qi in China, a new railway bridge with a span of more than $64 \mathrm{~m}$ but a construction depth of less than $2.4 \mathrm{~m}$ will be built. A railway in a certain place will also pass through the bridge. Therefore, a concrete-filled steel tube arch bridge with a slot beam is selected. For this new structure that is rarely used in engineering, there is no research result on the mechanical properties of the structure. Therefore, he designed an experimental model with a geometric ratio of $1: 4$ for the bridge to test the mechanical properties under operating load, crack load, and ultimate load (such as crack diagram, bearing capacity, stress distribution, and failure mechanism). According to the experimental results, when the model is subjected to a longitudinal bending moment, the failure mode is similar to the pure bending of the beam; when the model is subjected to a lateral bending moment, the failure mode behaves similar to the bending failure mode. The model is also analyzed by nonlinear elements, and the results are in good agreement with the experimental results. Considering the various loads in the design, the same modeling method was applied to the finite element analysis of the real bridge. After analysis, he finally confirmed that this new type of bridge meets the specifications for strength, stiffness, and crack resistance [8]. Chen believes that the construction of transportation infrastructure has a profound impact on the coordinated development of the regional economy and urban agglomerations. The completion of the bridge can meet the growing regional traffic demand and change the regional spatial traffic pattern, thereby enhancing the location advantage of the west coast of the region, promoting urban integration and economic development, and achieving coordinated urban development [9]. Wei et al. put forward a new design idea of the inner cone flow field of the inward rotating inlet. The basic flow field is designed by the characteristic rotation method, including two shock waves, a leading-edge shock wave, and an end shock wave. There is no continuous impact reflection along the internal flow path. Their numerical simulation results show that the incident positions of the two shock waves fully meet the design requirements, and there is no shock wave reflection at the shoulder of the inlet. The distribution of Mach numbers along the slope obtained by the feature rotation method is in good agreement with the CFD results [10]. The flow parameters at the throat are almost uniform, with a total pressure recovery factor of 0.91 . In the nonviscous condition, the entry of flow tracking completely inherits the basic flow field characteristics. The flow capture factor is 0.999 . The total pressure recovery coefficient is 0.88 , which is almost equal to the value of the inlet of flow tracking in the same design [11].

In this paper, a numerical simulation of the dynamic response of a breakwater against waves is carried out. The overall structure model of the breakwater was established by the dynamic contact method, and the fluid part was modeled based on the ALE method, and the fluid-solid coupling was achieved by using the penalty function method. The reliability of the method was verified through experiments, and the numerical analysis was used to further analyze the impact of wave pressure distribution, the maximum horizontal thrust on the wave wall during the impact process, and the dynamic response of the structure on the wave impact coefficient. The work outline of this paper is as follows: (1) Based on the collection of a large amount of research data on the wave (flow) forces of marine structures, combined with the research data on the foundation wave currents of existing sea-crossing bridges, the current status of the research on bridge wave forces is summarized and analyzed. Aiming at the problems of current wave force calculation of the foundations of cross-sea bridges, the key points and research ideas for further research are put forward. (2) Based on the cross-Qiongzhou Strait project, a three-dimensional wave and ocean current mathematical model is established to calculate the wave current forces under different structures and different conditions in the Qiongzhou Strait. Based on the analysis of the calculation results, the foundation of the cross-sea bridge is initially analyzed, and the change characteristics of wave (current) force are obtained. (3) Through the wide flume wave model test, the forces of the bridge foundation and the construction cofferdam under the combined action of regular wave action, irregular wave action, tidal action, and wave current are systematically studied.

\section{Proposed Method}

2.1. Cloud Computing. Due to the continuous decline in the cost of computing core components brought about by the development of large-scale integrated circuits, the first personal computer finally came out in 1969 , and computers began to become civilians to meet people's computing needs. When the third major change in IT technology was when the Internet was born, it was also when people's demand for information broke out. People hope to transmit and exchange information at a faster speed. At this time, relying solely on their own information processing capabilities and inability to meet people's requirements for information volume, they need to cooperate to communicate to meet the requirements for obtaining information. When the communication cost is further reduced and the communication coverage and communication speed are further increased, what people want to obtain is pure information without any 
other hardware [12]. At this time, service software, etc., information processing services have begun to emerge, and cloud computing as the implementation technology of this concept will surely flourish into a new innovation in IT technology [13].

Cloud computing is a supercomputing model based on the Internet. It distributes computing tasks on a resource pool composed of a large number of computers, enabling various application systems to obtain computing power, storage space, and various software services as needed. Cloud computing essentially accesses applications and services through the Internet, and these applications or services are usually not run on their own servers, but are provided by third parties. Its goal is to get everything to the network. The cloud is the network, and the network is the computer. The novelty of cloud computing is that it can provide almost unlimited cheap storage and computing power [14].

Cloud computing ultimately brings more convenience, so from the user's perspective, cloud computing has its unique new attraction [15]. At present, it is generally believed that cloud computing should have the following characteristics.

(1) Super Large Scale. "Cloud" has a considerable scale. Google Cloud Computing has more than 1 million servers. Amazon, IBM, Microsoft, 1100, and other "clouds" all have hundreds of thousands of servers. Enterprise private clouds typically have hundreds or thousands of servers. "Cloud" can give users unprecedented computing capabilities [16, 17]. (2) Virtualization. Cloud computing supports users to obtain application services at any location and using various terminals. The requested resource comes from the "cloud," not a fixed tangible entity. Applications run somewhere in the "cloud," but users do not actually need to know or worry about where the application runs. Only a laptop or a mobile phone is needed to achieve everything we need through network services, including tasks such as supercomputing. (3) High Reliability. "Cloud" uses measures such as multicopy fault tolerance of data and homogeneous interchangeability of computing nodes to ensure high reliability of services. Using cloud computing is more reliable than using local computers. (4) Versatility. Cloud computing does not target specific applications. With the support of the "cloud," it is possible to construct ever-changing applications. The same "cloud" can support different application operations at the same time. (5) High Scalability. The scale of the "cloud" can be dynamically scaled to meet the needs of application and user growth [18]. (6) On-Demand Services. "Cloud" is a huge resource pool, you can buy it on demand; cloud can be charged like tap water, electricity, and gas. (7) Extremely Cheap. Because the special fault tolerance measures of "cloud" can use extremely cheap nodes to form the cloud, the automated centralized management of "cloud" saves a large number of enterprises from having to bear increasingly high data center management costs, and the universality of "cloud" enables resource utilization compared with the traditional system; it is greatly improved, so users can fully enjoy the low-cost advantage of the "cloud" [19]. Often, it only takes hundreds of dollars and a few days to complete tasks that previously required tens of thousands of dollars and months to complete.

\subsection{Mathematical Model}

2.2.1. Mass Conservation Equation. The meaning of the continuity equation is that the mass of the fluid contained in a fluid system remains unchanged during the movement. In other words, the rate of fluid mass reduction in a fixed space is equal to the mass flux through its surface during this time [20]. The former is for the system and the latter is for the control body. Use this law as a mathematical expression-a continuity equation:

$$
\frac{\partial \rho}{\partial t}+\frac{\partial(\rho u)}{\partial x}+\frac{\partial(\rho v)}{\partial y}+\frac{\partial(\rho w)}{\partial z}=0
$$

where $\rho$ is the density of the fluid; $u$ is the velocity component in the $x$-direction, $v$ is the velocity component in the $y$-direction, and $w$ is the velocity component in the $z$-direction. The N-S equation can describe the actual fluid motion more accurately.

2.2.2. Momentum Conservation Equation. The momentum equation (N-S equation) of constant viscosity incompressible fluid can be used to accurately describe the actual fluid motion. The equations in the $x$-, $y$-, and $z$-directions are

$$
\begin{array}{r}
\rho\left(\frac{\partial u}{\partial t}+u \frac{\partial u}{\partial x}+v \frac{\partial u}{\partial y}+w \frac{\partial u}{\partial z}\right)=\rho F_{x}-\frac{\partial \rho}{\partial x}+\mu\left(\frac{\partial^{2} u}{\partial x^{2}}+\frac{\partial^{2} u}{\partial y^{2}}+\frac{\partial^{2} u}{\partial z^{2}}\right) \\
\rho\left(\frac{\partial v}{\partial t}+u \frac{\partial v}{\partial x}+v \frac{\partial v}{\partial y}+w \frac{\partial v}{\partial z}\right)=\rho F_{x}-\frac{\partial \rho}{\partial y}+\mu\left(\frac{\partial^{2} v}{\partial x^{2}}+\frac{\partial^{2} v}{\partial y^{2}}+\frac{\partial^{2} v}{\partial z^{2}}\right) \\
\rho\left(\frac{\partial w}{\partial t}+u \frac{\partial w}{\partial x}+v \frac{\partial w}{\partial y}+w \frac{\partial w}{\partial z}\right)=\rho F_{x}-\frac{\partial \rho}{\partial z}+\mu\left(\frac{\partial^{2} w}{\partial x^{2}}+\frac{\partial^{2} w}{\partial y^{2}}+\frac{\partial^{2} w}{\partial z^{2}}\right)
\end{array}
$$

2.2.3. Turbulence Model. The $k-\varepsilon$ model is selected for the turbulence model in this paper. The turbulent kinetic energy is $k$ and the dissipation energy is $\varepsilon$. The calculation equation is 


$$
\begin{aligned}
& \rho \frac{d k}{d t}=\frac{\partial}{\partial x_{i}}\left[\left(\mu+\frac{\mu_{t}}{\sigma_{k}}\right) \frac{\partial k}{\partial x_{i}}\right]+G_{k}+G_{b}-\rho \varepsilon-Y_{M}, \\
& \rho \frac{d \varepsilon}{d t}=\frac{\partial}{\partial x_{i}}\left[\left(\mu+\frac{\mu_{t}}{\sigma_{\varepsilon}}\right) \frac{\partial \varepsilon}{\partial x_{i}}\right]+C_{1 \varepsilon} \frac{\varepsilon}{k}\left(G_{k}+C_{3 \varepsilon} G_{b}-C_{2 \varepsilon} \rho \frac{\varepsilon^{2}}{k}\right),
\end{aligned}
$$

where $G_{k}$ is the turbulent flow energy generated by the average velocity gradient; $G_{b}$ is the turbulent flow energy generated by the buoyancy effect; and $\mu_{t}=\rho C_{\mu} k^{2} / \varepsilon$ is the effect of turbulent pulsation expansion on the total dissipation rate.

2.2.4. Free Surface Tracking Equation. Due to the continuity of the fluid, the fluid can be regarded as a continuous medium composed of numerous units, and each physical quantity of the fluid can be described by space coordinates and time functions. By using the VOF model to calculate the continuous equation of the volume fraction of each element between each phase, the free surface of a multiphase mixed fluid can be monitored. Its continuous equation is

$$
\frac{\partial a_{q}}{\partial t}+v_{q} \nabla a_{q}=\frac{S_{a q}}{\rho_{q}}+\frac{1}{\rho_{q}} \sum_{p=1}^{n}\left(\dot{m}_{p q}-\dot{m}_{q p}\right),
$$

where $m_{p q}$ is the mass transferred from phase $p$ to phase $q$, $a_{q}=0$ indicates that the unit $a$ of phase $q$ is empty and there is no fluid therein; $a_{q}=1$ indicates that the unit is completely filled with fluid; and $0<a_{q}<1$ indicates that the unit is a free surface unit.

2.2.5. Wave Elimination Model. The moving boundary equation of motion along the $x$-direction is $x=A \sin (\omega t)$, where $A$ is the maximum stroke of the push plate. In the processing of the elimination band, the macro DEFINE_SOURCE (mom_source, cell: thread, dS, eqn) is used.

Its momentum equation can be rewritten as

$$
\frac{\partial u}{\partial t}+u \frac{\partial u}{\partial x}+\omega \frac{\partial u}{\partial z}=-\frac{1}{\rho} \frac{\partial \rho}{\partial x}+\mu\left[\frac{\partial^{2} u}{\partial x^{2}}+\frac{\partial^{2} u}{\partial z^{2}}\right]-\mu u .
$$

Among them, $\mu$ is the attenuation coefficient and $u$ is the speed component in the $x$-direction. When the wave propagates into the wave-cutting region, the wave energy equation is subtracted by one $\mu u$ after each iteration, so that the wave-cutting purpose is achieved after $n$ iterations.

As shown in Figure 1, when the solver solves, it solves the above continuous, momentum, and energy equations, respectively, and finally obtains the solutions of all the equations. When solving the accompanying scalar equations, such as calculating the turbulence model and the wave elimination equation, separate solution is used; that is, the governing equation is solved first, and then the turbulence model equation and the wave elimination equation are solved [21].

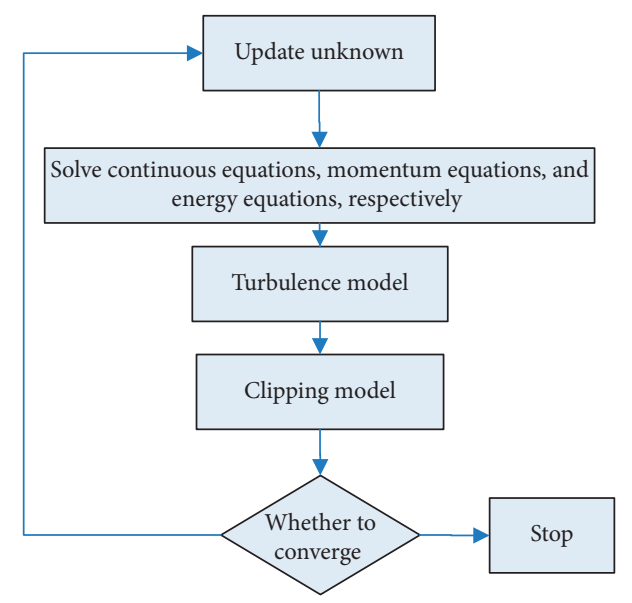

FIGURE 1: Calculation flowchart.

2.3. Fluent Introduction. Fluent is a general-purpose CFD software calculation package that can perform a large number of complex process simulations, including a wide range from incompressible to compressible, from low speed to supersonic speed, and has been obtained in different fields such as engineering, business, and academics. It is widely used and can stand the test of practice $[22,23]$. At present, both the meshes generated by ICEM and Gambit can be used with Fluent, and both can meet the requirements of Fluent computing to the greatest extent [24].

The Fluent software package contains a variety of solvers, including separate solvers and coupled solvers, pressure and density basis, and difference format with display and implicit. For various types of complex flow fields that are incompressible to the ultrahigh sound speed range, targeted processing needs to be made. The choice of each type of problem solver is different, and the use of the solver can make Fluent widely used. In terms of physical models, Fluent software is rich in types and has been applied in the field of engineering. The reliability of the results has been verified. In terms of convergence speed and solution accuracy, due to the use of multiple solution methods and multigrid accelerated convergence techniques in the solution process, the best accuracy can be achieved $[25,26]$. It is precisely because Fluent has accurate physical models, free unstructured grids, and solution-based adaptive grid technology that can simulate complex flow problems of mechanisms and physical processes, such as hypersonic flow fields, heat transfer and phase transitions, chemical reaction and combustion, multiphase flow, rotating machinery, moving/ deformed grids, noise, material processing, and other problems or a mixture of multiple physical problems. The solution of Fluent software relies on the finite volume method, uses a completely unstructured mesh, and has a gradient algorithm based on mesh nodes and mesh elements [27].

Fluent software also has a variety of grid-based technologies. Based on the results of the solution, grid adaptation can be performed, or the two technologies can be used in 
combination, which is efficient, time-saving, and fast. Computing software in different fields is organically linked with Fluent to form a CFD computer software group [28]. With Fluent as a bridge tool, the value exchange between different software is faster and more accurate. The unification of preprocessing and postprocessing tools, for scientific researchers, can focus on the nature of the physical problems and phenomena being studied, instead of spending a lot of time thinking about computing methods, grid preprocessing, programming, postprocessing processing, and other tasks; more time is spent in areas that require more scientific researchers. Based on the flow and physical characteristics of different problems, users can independently choose a numerical solution that meets the requirements and choose to display or implicit difference format, so that the calculation process can achieve the expected results in terms of accuracy, speed, and stability $[29,30]$. Fluent has performed simulation calculations on a large number of engineering examples. The simulation results are in good agreement with the actual results and have a high degree of persuasion. The accuracy of the calculation results can reach the second-order finite volume method, also known as control volume law. In computational fluid dynamics, it is mainly used to solve the problems of fluid flow and heat transfer. The finite volume method describes each control body defined by the computational grid, and the solution process is based on the conservation equations of the integral form, not the differential equations. For the integral conservation equation, a discrete equation is constructed from a physical point of view. Each expression of the conservation of physical quantities on the control volume represents a discrete equation. The physical derivation process based on this method can clearly reflect the physical concept, the coefficient of the discrete equation expresses a certain physical meaning, and the conservation meaning of the discrete equation is also guaranteed. Finite volume method solution ideas and steps are as follows:

(1) Divide the computational domain to generate nonrepeating control bodies. The control nodes are represented by grid nodes, and the differential equations are based on time and space integration in the control bodies and time intervals.

(2) Put forward the hypothesis, and choose a suitable line or interpolation method of the function and its derivative for time and space.

(3) According to the selected profile and interpolation method, each control body in 1 is integrated, and a set of discrete equations about the unknowns on the nodes can be obtained by sorting.

\section{Experiments}

3.1. Hydrogeological Conditions at the Bridge Site. The water depth near the coast of the bridge is relatively shallow, most of the area is $1 \sim 2 \mathrm{~m}$, and the deepest water depth is about
$6 \mathrm{~m}$. The maximum water depth in the main channel area is approximately $25 \mathrm{~m}$. The major threats to the bridges are mainly offshore waves, which basically propagate from the area between the SE-SSE directions. With reference to local measured data for many years, the average wind speed of the bridge is $6.9 \mathrm{~m} / \mathrm{s}$, the maximum wind speed is $34 \mathrm{~m} / \mathrm{s}$, the normal wind direction is NNE, and the frequency is $43 \%$. The annual average wind level of 107.2 days is not less than level 8 , and the maximum can reach 172 days.

3.2. Experimental Design Wave Elements. According to the geographical location of the bridge and the hydrometeorological conditions of the bridge, the design conditions of the wave and water flow elements (horizontal bridge direction) are to be selected once in 98 years, once in 48 years, and once in 18 years. The design wave elements are shown in Table 1.

3.3. Establishment of Numerical Model. As shown in Table 1, this article uses Fluent 12.0 to establish a numerical wave flume and generates a model of the interaction between the main beam and the different impact heights of the wave. The waves are calculated numerically. Based on the obtained time-history curves of the total wave force coefficients acting on the bridge girder in each model, the magnitude of the wave force on each main beam is further calculated, and the simulation results are compared and analyzed. The main beam of the bridge is a cross section of a box girder. The width of the top of the box girder is $17 \mathrm{~m}$, the width of the bottom is $9 \mathrm{~m}$, and the height is $3.5 \mathrm{~m}$. The cross section of the bridge is shown in Figure 2. Taking into account the possible height of the main beam of the bridge and the actual height of the box beam of $3.5 \mathrm{~m}$, the wave conditions at high water levels are designed according to the three working conditions, and the side heights of the main beam of the wave impact are $0.5,1.0$, and 1.5 , seven calculation models including 2.0, 2.5, 3.0, and $3.5 \mathrm{~m}$. The water level depths are 10,15 , and $21 \mathrm{~m}$. A total of 63 models need to be established.

According to general modeling principles, the proportion of the length of the main beam model in the entire numerical wave flume is best kept at about $1: 8$ to $1: 10$. It can ensure that the waves can fully develop without wasting calculation time and computer space because the wave tank is too large. The position of the bridge model in the wave tank is determined comprehensively according to factors such as model size and wave wavelength. It is best to ensure that there are 1-2 fully developed wavelengths in front of the main beam, and there is sufficient wave elimination and buffer area behind the main beam.

According to the width of the bridge deck, take about 10 times the width of the bridge deck, that is, $180 \mathrm{~m}$ as the length of the wave trough. The length in front of the main beam in the wave trough is $100 \mathrm{~m}$, which can contain 1 to 2 fully developed wavelengths. The area behind the main beam is $117 \sim 180 \mathrm{~m}$. This size can ensure that after the waves in the numerical wave flume impact the bridge's main beam, there is 
TABle 1: Table of design wave elements.

\begin{tabular}{lcccccc}
\hline $\begin{array}{l}\text { Wave } \\
\text { element }\end{array}$ & $\begin{array}{c}\text { Water level } \\
(\mathrm{m})\end{array}$ & $\begin{array}{c}\text { Water depth } \\
(\mathrm{m})\end{array}$ & $\begin{array}{c}\text { Feature point } H_{1 \%} \\
(\mathrm{~m})\end{array}$ & $\begin{array}{c}\text { Mean wave height } \bar{H} \\
(\mathrm{~m})\end{array}$ & $\begin{array}{c}\text { Wave height } \bar{T} \\
(\mathrm{~m})\end{array}$ & Wavelength $L(\mathrm{~m})$ \\
\hline 98 years & +5.2 & 22.6 & 5.7 & 2.5 & 5.6 & 66.0 \\
48 years & +5.1 & 21.5 & 5.1 & 2.3 & 5.1 & 5.5 \\
18 years & +4.8 & 20.2 & 4.2 & 1.9 & 4.0 & 46.9 \\
\hline
\end{tabular}

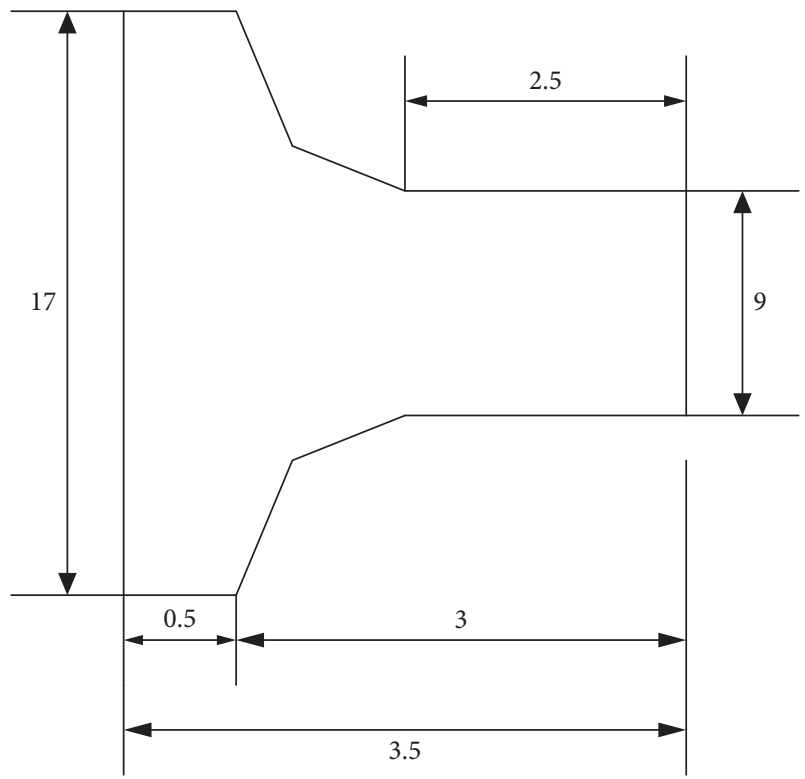

Figure 2: Schematic diagram of bridge cross section.

a sufficient buffer when flowing behind the main beam, so that there is no wave reflection due to the insufficient buffer length. The height of the free air in the upper part of the main beam can be $15 \mathrm{~m}$, and the depth of the water flow in the wave flume is different depending on the simulated working conditions.

In this study, the Fluent VOF method was used to solve the free surface of the water-gas boundary in a wave flume. The flow field was calculated using the segregated, standard $k-\varepsilon$ model and the pressure-speed coupled PIOS algorithm. The boundary conditions in the Fluent VOF model are set as follows: the bottom is set to the wall boundary condition; the upper boundary is set to the symmetric boundary (symmetry). When it is set to the symmetric boundary type, there is no exchange of mass and momentum inside and outside this boundary. Facilitate rapid convergence of the calculation; the left and right boundaries are the wave and air flow boundary and the outflow boundary, respectively, with the water surface as the interface, and the left and right boundaries are set as the velocity-inlet and outflow boundaries, respectively.

\section{Discussion}

4.1. Fluent Numerical Analysis of Lifting Resistance and Wave Impact Height of a Sea-Crossing Bridge

4.1.1. Iterative Calculation Data Analysis. After the model is read in, the wave speed is determined according to the wave length and period of the model. After inputting the parameters such as the wavelength and wave height, the iterative calculation is performed one by one. During the calculation process, the development time of the lift coefficient and drag coefficient of the wave is mainly monitored. In order to obtain the state of maximum force, generally 2 to 3 cycles are calculated. The iterative calculation time interval is set to $0.001 \mathrm{~s}$, and iterative calculation is performed 20 times in each time interval. In order to prevent the phenomenon of excessive numerical distortion due to the instability of the model calculation at the initial moment of calculation, the author performs a preliminary trial calculation for a short period of time before setting the rising resistance monitoring curve to monitor the convergence of the residual error to make the calculation error controlled within a reasonable range. After the convergence of the residuals is stable, iterative calculation of the model is performed, and the lift resistance coefficient is monitored. When the model residuals converge, as shown in Figure 3, when the iterative calculation is about 100 times, the calculation error is basically stable, and then the numerical simulation calculation of the model can be performed.

4.1.2. Analysis of Lift Coefficient and Drag Coefficient at Different Water Depths in the case of 20 Years. As shown in Table 2 and Figure 4, the law of the wave lift resistance coefficient changes with the height of the wave impacting the main beam under the conditions of 20 years. The figure also shows the change law of the lift resistance coefficient at different water depths under the same wave factor. The curve of the lift resistance coefficient corresponding to different water depths is basically the same. When the wave impact height is $0.5 \sim 2.0 \mathrm{~m}$, the coefficient gradually increases, and a small peak point appears at $2.0 \mathrm{~m}$; the impact height is smaller at about $2.5 \mathrm{~m}$. The degree of fallback occurred at $3.0 \mathrm{~m}$. This shows that when the wave impact height is 2.0 and $3.0 \mathrm{~m}$, the wave force experienced by the bridge is large, as shown in Table 3. Therefore, we should focus on the wave forces with wave impact heights of 2.0 and $3.0 \mathrm{~m}$. It can also be seen that when the wave impact height is $2.0 \mathrm{~m}$ and below, the lifting resistance coefficients of the three water depths can maintain a high consistency, and the numerical difference between them is not large. After the impact height is higher than $2.0 \mathrm{~m}$, with the different water depth, there is a large inconsistency in the lift resistance coefficient, especially at the depth of $10 \mathrm{~m}$, when the impact height is 3.0 and $3.5 \mathrm{~m}$, it is different from the other two water depths. There is a clear gap in drag coefficients.

4.1.3. Analysis of Lift Coefficient and Drag Coefficient in Different Water Depths in 50 Years. As shown in Figure 5, it 


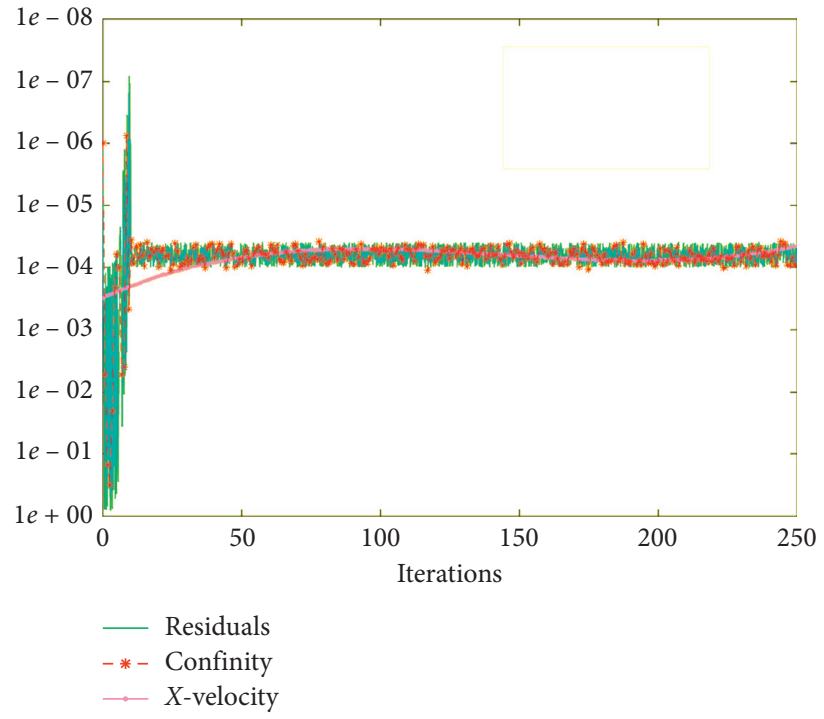

FIGURE 3: Simulation calculation residual plot.

TABLE 2: Statistics of lift coefficient of wave impact height.

\begin{tabular}{|c|c|c|c|c|c|c|c|}
\hline Wave impact height $(\mathrm{m})$ & 0.2 & 0.7 & 1.2 & 1.7 & 2.2 & 2.7 & 3.2 \\
\hline 10 & 15.6792 & 16.6792 & 20.6792 & 51.6792 & 34.6792 & 60.6792 & 38.6792 \\
\hline 15 & 14.6792 & 15.6792 & 19.6792 & 49.6792 & 31.6792 & 28.6792 & 21.6792 \\
\hline 21 & 11.6792 & 14.6792 & 18.6792 & 46.6792 & 16.6792 & 32.6792 & 22.6792 \\
\hline
\end{tabular}

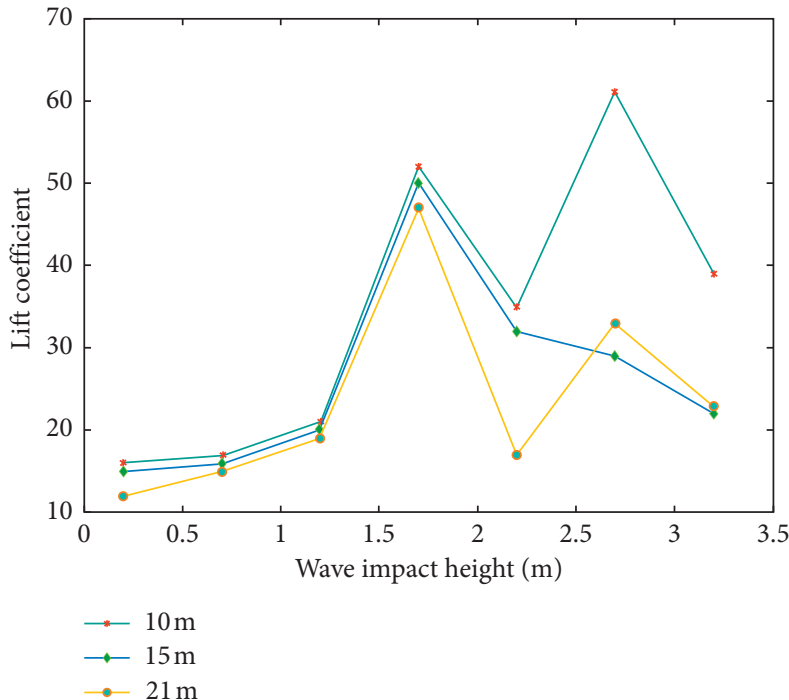

(a)

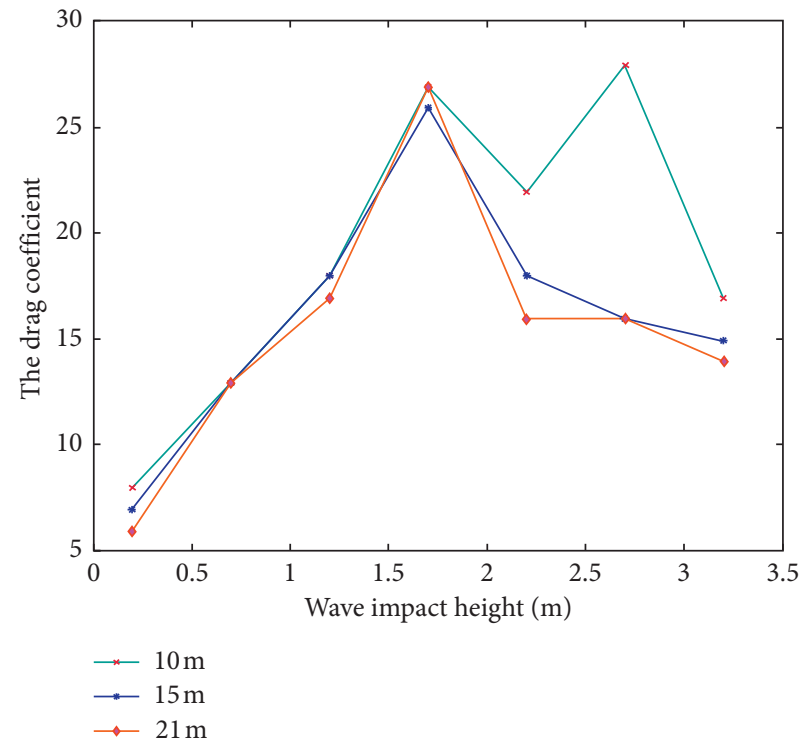

(b)

Figure 4: Analysis of the lift coefficient and drag coefficient in different water depths in case of working conditions. (a) Lift coefficient. (b) Resistance coefficient.

TABLE 3: 20 analysis of lift coefficient and resistance coefficient of different water depths in case of working conditions.

\begin{tabular}{lccccccc}
\hline Wave impact height $(\mathrm{m})$ & 0.2 & 0.7 & 1.2 & 1.7 & 2.2 & 2.7 \\
\hline 10 & 7.9348 & 12.9348 & 17.9348 & 26.9348 & 21.9348 & 27.9348 & 16.9348 \\
15 & 5.9348 & 12.9348 & 16.9348 & 26.9348 & 15.9348 & 15.9348 & 13.9348 \\
21 & 6.9348 & 12.9348 & 17.9348 & 25.9348 & 17.9348 & 15.9348 & 14.9348 \\
\hline
\end{tabular}




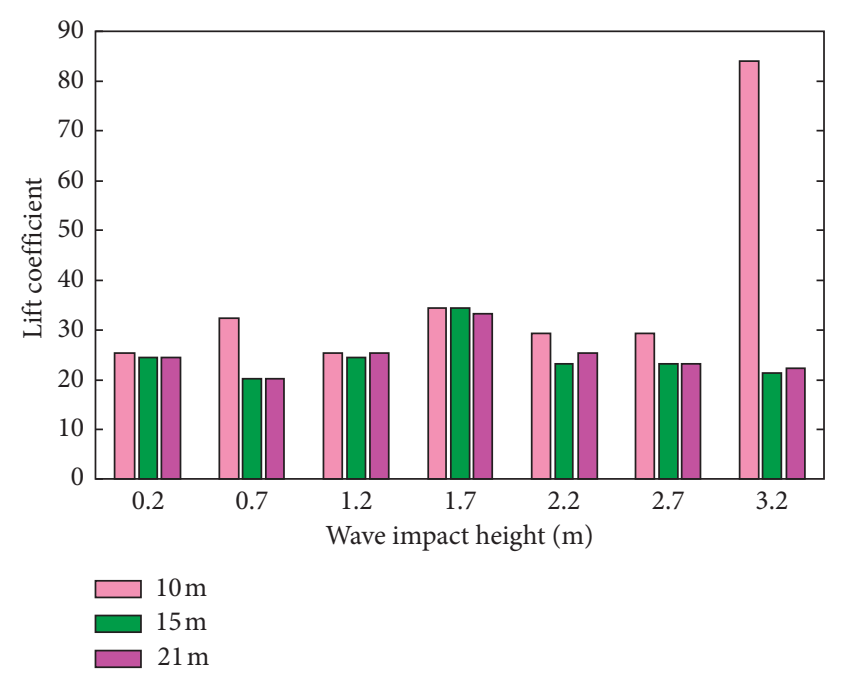

FIGURE 5: 50 lifting coefficient and resistance coefficient of different water depths in case of working conditions.

shows the change law of the wave lift resistance coefficient with the height of the wave impacting the main beam under the conditions of 50 years and the correlation between the lift resistance coefficient at different water depths. The change law of the lift resistance coefficient curve with water depth is basically similar to the once-in-a-year working conditions. The difference between the two conditions is that under different water depths, the difference in the 50-year rise resistance coefficient is greater than 20 years. The difference is mainly due to the more complicated wave conditions encountered once in 50 years than in the case encountered once in 20 years and the uncertainty of the wave force of the main beam. When the water depth is $10 \mathrm{~m}$, the curve of the lift coefficient is obviously different from the other two conditions.

The impact coefficient value of each section of the bridge generally decreased with the increase of vehicle speed, but there was no obvious law. Among them, the impact coefficient value of the secondary side span has no significant change with the increase of vehicle speed; the impact coefficient of the side span has the most dramatic change with the vehicle speed, and the changes of the secondary side span and the middle span are relatively gentle.

4.1.4. Lift Coefficient and Drag Coefficient Diagrams at Different Water Depths in the case of 100 Years. In Figure 6, the change law of the wave lift resistance coefficient with the wave impact on the height of the main beam under the 100year case is shown. When the water depth is $15 \mathrm{~m}$ and $21 \mathrm{~m}$, the curve shows a more stable trend, and no large peak point appears between the points in the curve.

The impact coefficients at different damping ratios at each position can be seen: the impact coefficient of the impact coefficient with the vehicle speed changes at the same position with different damping ratios. The bridge damping ratio has a more obvious impact on the impact coefficient, and the larger the damping ratio, the smaller the impact coefficient, and the impact on the impact coefficient of

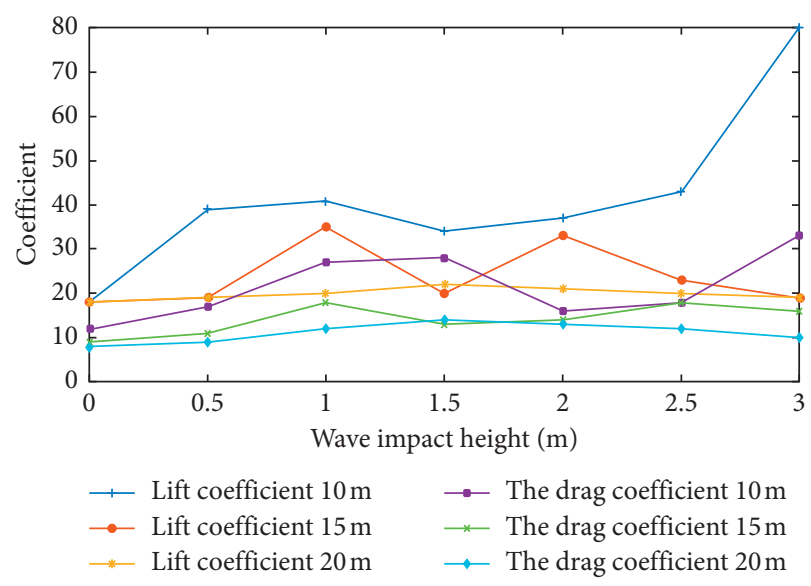

Figure 6: 100 lifting coefficient and drag coefficient in different water depths.

different parts is different. Comparing the change curve of the lift resistance coefficient under working conditions, it can be seen that there are two values for the height of the main beam where a large wave force is applied, 2.0 and $3.0 \mathrm{~m}$ in the case of 20 years; once in the case of 50 years at 2.5 and $3.0 \mathrm{~m}$; the lift coefficient is 1.5 and $3.5 \mathrm{~m}$, and the resistance coefficient is 2.0 and $3.5 \mathrm{~m}$. Under the three water depth wave conditions, the fluctuation range of the lifting resistance curve is large when the water level depth is $10 \mathrm{~m}$. Once a large wave occurs, the wave will not spread well due to the shallow current, which will cause a large wave force impact on the bridge. Therefore, when selecting the bridge site, it is not recommended to build at a depth of about $10 \mathrm{~m}$.

\section{Conclusions}

Based on cloud computing technology, this paper establishes a numerical monitoring model for the lifting resistance and wave impact height of the cross-sea bridge. Use the basic test data of the established cross-sea bridge to verify the model; use the verified three-dimensional mathematical model, select typical structures as the research object, calculate the wave force under different working conditions, and analyze the initial wave force of the cross-sea bridge. The characteristics of force change. The results of the next stage tank model test are used to verify and improve the mathematical model, and finally the three-dimensional wave current calculation mathematics suitable for caisson, roof, and pier is proposed.

When the traditional finite element model is established in this paper, usually due to the idealized assumptions on the material parameters, component connection methods and boundary conditions of the structure, and due to the differences in the construction process, the calculated response and the measured response of the model established deviations, so it is necessary to modify the established finite element model to find the basic finite element model of the structure.

In this paper, the wave impact coefficients of wave barriers with different structural stiffness at the maximum horizontal wave impact force on front and rear wave barriers 
under different hydrological conditions are obtained through numerical calculations. The maximum stress of the back wall structure in the design condition is about twice the front wall; the rear wave wall always bears larger horizontal forces than the front wave wall; the dynamic response of the structure has an amplification effect on the wave impact coefficient, and the structure is with small stiffness.

\section{Data Availability}

No data were used to support this study.

\section{Conflicts of Interest}

The authors declare that they have no conflicts of interest regarding the publication of this paper.

\section{Acknowledgments}

This work was supported by the open fund of State Key Laboratory of Mountain Bridge and Tunnel Engineering (SKLBT-19-005).

\section{References}

[1] S. Dustdar, "Cloud computing," Computer, vol. 49, no. 2, pp. 12-13, 2016.

[2] S. Bera, S. Misra, J. J. P. C. Rodrigues, and P. C. Rodrigues, "Cloud computing applications for smart grid: a survey," IEEE Transactions on Parallel and Distributed Systems, vol. 26, no. 5, pp. 1477-1494, 2015.

[3] Y. Wang, J. Li, and H. H. Wang, "Cluster and cloud computing framework for scientific metrology in flow control," Cluster Computing, vol. 22, no. 1, pp. 1-10, 2019.

[4] D. Boru, D. Kliazovich, F. Granelli, P. Bouvry, and A. Y. Zomaya, "Energy-efficient data replication in cloud computing datacenters," Cluster Computing, vol. 18, no. 1, pp. 385-402, 2015.

[5] S. Liu, Y. Yang, W. G. Qu, and Y. Liu, "The business value of cloud computing: the partnering agility perspective," Industrial Management \& Data Systems, vol. 116, no. 6, pp. 1160-1177, 2016.

[6] M. Anshari, Y. Alas, and S. G. Lim, "Pervasive knowledge, social networks, and cloud computing: e-learning 2.0," Eurasia Journal of Mathematics Science \& Technology Education, vol. 11, no. 5, pp. 909-921, 2015.

[7] S. Namasudra and P. Roy, "PpBAC: popularity based access control model for cloud computing," Journal of Organizational and End User Computing, vol. 30, no. 4, pp. 14-31, 2018.

[8] M. Grujicic, J. Snipes, and S. Ramaswami, "A computational analysis of the utility of chemical reactions within protective structures in mitigating shockwave-impact effects," Multidiscipline Modeling in Materials and Structures, vol. 12, no. 3, pp. 438-472, 2016.

[9] Z. Chen and Jinan University, "Cross-sea passage and coordinated development of city group: Zhuhai-Macao Bridge as an example," Science \& Technology Review, vol. 34, no. 21, pp. 64-68, 2016.

[10] F. Wei, X.-Z. He, and Y.-Y. He, "Design method of dual-shock wave basic flow-field for inward turning inlet," Journal of Propulsion Technology, vol. 36, no. 3, pp. 358-364, 2015.

[11] T. Wang, Z. Zheng, and M. Elhoseny, "Equivalent mechanism: releasing location data with errors through differential privacy," Future Generation Computer Systems, vol. 98, pp. 600-608, 2019.

[12] Z. Cao, L. Jin, and C. Wan, "Optimal cloud computing resource allocation for demand side management in smart grid," IEEE Transactions on Smart Grid, vol. 8, no. 4, pp. 1943-1955, 2017.

[13] T. Kaur and I. Chana, "Energy efficiency techniques in cloud computing-a survey and taxonomy," ACM Computing Surveys, vol. 48, no. 2, pp. 1-46, 2015.

[14] J. Wang, X. Chen, and X. Huang, "Verifiable auditing for outsourced database in cloud computing," IEEE Transactions on Computers, vol. 64, no. 11, p. 1, 2015.

[15] L. Fabisiak, "Web service usability analysis based on user preferences," Journal of Organizational and End User Computing, vol. 30, no. 4, pp. 1-13, 2018.

[16] A. F. Barsoum and M. A. Hasan, "Provable multicopy dynamic data possession in cloud computing systems," IEEE Transactions on Information Forensics and Security, vol. 10, no. 3, pp. 485-497, 2015.

[17] S.-B. Tsai and K. Wang, "Using a novel method to evaluate the performance of human resources in green logistics enterprises," Ecological Chemistry and Engineering S, vol. 26, no. 4, pp. 629-640, 2019.

[18] O. C. Ann, F. S. Tee, V. Y. Nen, S. Fu, and V. Y. Nen, “A study on satisfaction level among amateur web application developers towards pigeon-table as nano web development framework," Journal of Organizational and End User Computing, vol. 31, no. 3, pp. 97-112, 2019.

[19] M. R. Watson, Noor-ul-hassan Shirazi, A. K. Marnerides, A. Mauthe, and D. Hutchison, "Malware detection in cloud computing infrastructures," IEEE Transactions on Dependable \& Secure Computing, vol. 13, no. 2, pp. 192-205, 2015.

[20] A. N. Tashkandi and I. M. Al-Jabri, "Cloud computing adoption by higher education institutions in Saudi Arabia: an exploratory study," Cluster Computing, vol. 18, no. 4, pp. 1527-1537, 2015.

[21] H. M. Sabi, F.-M. E. Uzoka, K. Langmia, and F. N. Njeh, "Conceptualizing a model for adoption of cloud computing in education," International Journal of Information Management, vol. 36, no. 2, pp. 183-191, 2016.

[22] W.-L. Shiau and P. Y. K. Chau, "Understanding behavioral intention to use a cloud computing classroom: a multiple model-comparison approach," Information \& Management, vol. 53, no. 3, pp. 355-365, 2015.

[23] J. Moura and D. Hutchison, "Review and analysis of networking challenges in cloud computing," Journal of Network and Computer Applications, vol. 60, no. 1, pp. 113-129, 2016.

[24] C. Colman-Meixner, C. Develder, and M. Tornatore, "A survey on resiliency techniques in cloud computing infrastructures and applications," IEEE Communications Surveys \& Tutorials, vol. 18, no. 3, p. 1, 2016.

[25] U. Wajid, C. Cappiello, P. Plebani et al., "On achieving energy efficiency and reducing $\mathrm{CO}_{2}$ Footprint in cloud computing," IEEE Transactions on Cloud Computing, vol. 4, no. 2, pp. 138-151, 2016.

[26] C. Wang, K. Ren, and J. Wang, "Secure optimization computation outsourcing in cloud computing: a case study of linear programming," IEEE Transactions on Computers, vol. 65 , no. 1, pp. 216-229, 2016.

[27] D. S. Dolling, "On upstream influence in shock wave turbulent boundary layer interaction," Aeronautical Journal, vol. 87, no. 868, pp. 324-327, 2016.

[28] A. Haris, H. P. Lee, T. E. Tay, and V. B. C. Tan, "Shear thickening fluid impregnated ballistic fabric composites for 
shock wave mitigation," International Journal of Impact Engineering, vol. 80, no. 12, pp. 143-151, 2015.

[29] J. Holfeld, C. Tepeköylü, C. Reissig et al., "Toll-like receptor 3 signalling mediates angiogenic response upon shock wave treatment of ischaemic muscle," Cardiovascular Research, vol. 109, no. 2, p. 331, 2015.

[30] X. Wu, G. Shen, X. Gui, Z. Ling, and W. Chen, "Experimental and analytical behavior of a new type of concrete-filled steel tube tied-arch bridge with trough girder," Stahlbau, vol. 87, no. 3, pp. 256-267, 2018. 